\title{
Aetiology of Infectious Conjunctivitis- A Prospective Study
}

\author{
Chinnu R. S. ${ }^{1}$, Liby Joseph ${ }^{2}$ \\ ${ }^{1}$ Junior Resident, Department of Ophthalmology, Government Medical College, Kottayam, Kerala, India. \\ ${ }^{2}$ Associate Professor, Department of Ophthalmology, Government Medical College, Kottayam, Kerala, India.
}

\section{ABSTRACT}

\section{BACKGROUND}

Conjunctivitis is inflammation of the conjunctiva and is the most common cause of red eye. It may be due to infective causes or due to non-infective causes. The infective causes include those caused by bacteria and viruses. It may affect one or both eyes. Patients usually complain of redness, swelling and discharge from the eye. The type of discharge usually suggests the aetiology. It may be watery in viral conjunctivitis while purulent or muco-purulent in bacterial conjunctivitis. In this study, we have tried to confirm whether the clinical suspicion of a bacterial cause is actually bacterial by confirming with culture studies and to analyse which bacteria are commonly responsible for the infection.

\section{METHODS}

A prospective Study of 100 consecutive cases of conjunctivitis cases attending the Ophthalmology Outpatient Department were clinically evaluated and the conjunctival discharge was sent for bacterial culture. The results were correlated clinically and analysed using SPSS 16.0 version.

\section{RESULTS}

The study identified bacteria as the etiological agents in $20 \%$ of cases, of which $19 \%$ were coagulase negative Staphylococci and 1\% was Staphylococcus aureus. The rest of the $80 \%$ cases might include both viral and of unknown aetiology.

\section{CONCLUSIONS}

Majority of the patients clinically suspected to have bacterial conjunctivitis were confirmed to have a bacterial cause. This shows that a high index of clinical suspicion helps in giving correct treatment to the patients and they are spared from spending unnecessarily on culture studies. Thus, decreasing the economic burden of treating a common eye disease.

\section{KEY WORDS}

Conjunctivitis, Culture and Sensitivity, Conjunctival Discharge
Corresponding Author:

Dr. Liby Joseph,

Associate Professor,

Department of Ophthalmology,

Government Medical College,

Kottayam, Kerala, India.

E-mail: libydoctor@gmail.com

DOI: $10.14260 / \mathrm{jemds} / 2019 / 590$

Financial or Other Competing Interests: None.

How to Cite This Article:

Chinnu RS, Joseph L. aetiology of infectious conjunctivitis- a prospective study. J. Evolution Med. Dent. Sci. 2019;8(35): 2719-2723, DOI:
Submission 11-05-2019,

Peer Review 13-08-2019,

Acceptance 20-08-2019,

Published 02-09-2019. 


\section{BACKGROUND}

Inflammation or infection of the conjunctiva is known as conjunctivitis and is characterised by dilatation of the conjunctival vessels, resulting in hyperaemia and oedema of the conjunctiva, typically associated with discharge. Conjunctiva may be infected by bacteria, fungi, parasites, or viruses. Apart from the resident flora, any micro-organism from the environment can form a transient flora in the eye and given an opportunity invades the ocular tissues. The type of eye discharge and ocular symptoms can be used to determine the cause of the conjunctivitis. For example, a purulent or muco-purulent discharge is often due to bacterial conjunctivitis, whereas a watery discharge is more characteristic of viral conjunctivitis. However, the clinical presentation is often nonspecific. Relying on the type of discharge and patient symptoms does not always lead to an accurate diagnosis. Furthermore, scientific evidence correlating conjunctivitis signs and symptoms with the underlying cause is often lacking. Accurate knowledge about the possible etiological agent helps in avoiding prescription of unnecessary topical antibiotics or antiviral drugs. Thus, we may prevent the occurrence of antimicrobial resistance. In addition, the number of patients with drug side effects and the financial burden for treatment can also be markedly reduced.

We wanted to find out the aetiology of infectious conjunctivitis in patients coming to Ophthalmology OPD, during a one-year period, by culture studies.

\section{METHODS}

\section{Study Design}

Prospective study.

\section{Sample Size}

100.

\section{Inclusion Criteria}

Patients coming to Ophthalmology OPD with symptoms of infectious conjunctivitis, who have not taken any treatment for the condition.

\section{Exclusion Criteria}

1.Cases presumed to be allergic conjunctivitis with history of recurrent episodes \& associated systemic allergic conditions.

2.Patients with chemical or thermal or mechanical injury to eye

3.Patients with muco-cutaneous disease like bullous pemphigoid or Steven-Johnson syndrome

4.Patients who have taken prior treatment for the present condition

5.Patient not willing to be included in the study.

\section{Study Procedure}

Informed consent was obtained. Each patient included in the study was asked in detail about history of the illness and thorough clinical examination was done. Socio-demographic factors were noted. Patient was asked about history of recent or at present respiratory tract illness or fever, history of exposure to conjunctivitis cases within a period of 2 weeks prior to the beginning of symptoms. They were asked about any history of pre-existing diabetes mellitus \& history of immunosuppressive conditions like any malignancy, HIV infection, pregnancy, any history of organ transplantation or history of taking immunosuppressive drugs. History of blurring of vision after the illness started was also asked. Clinical features like unilateral or bilateral involvement, lid oedema, conjunctival congestion, follicular or papillary conjunctival reactions, chemosis, membranous or pseudo membranous reaction, type of discharge (Watery, mucoid or purulent type), sub-conjunctival haemorrhage, corneal involvement, ocular pain, uveitis, pre-auricular or sub mandibular lymph node enlargement were entered in the proforma. Conjunctival swabs were taken from each patient under strict sterile precautions by sweeping a sterile swab in the lower fornix of the patient without touching the eyelid margin. In patients with bilateral involvement the first affected eye was taken for sample collection. The sample was immediately sent to the Department of Microbiology for bacterial culture studies in a sterile bottle. There the swab was immersed in liquid glucose broth media and smeared on to blood agar, chocolate agar, MacConkey agar, salt agar and Robertson cooked meat media. It was kept overnight and looked for any organism growth on next day. If any plate showed organismal growth, it was further processed for identification of the organism. If the plate did not show any growth, the liquid glucose broth was further sub-cultured for the presence of any organism. If no organism was found out after 48 hours of incubation, the result was taken as sterile.

Second sample was transported to National Institute of Virology in Viral Transport Medium. The Viral transport media was obtained from the Institute of Virology. It was brought in cold chain method, kept in ice-packed container to the ophthalmology OPD. It was stored in the refrigerator. The swab taken from the patient was immersed in viral transport medium and the whole thing was stored in the freezer of the refrigerator. It could be stored in such a way up to one week without losing the potency of the sample. The sample was then transported to the institute of virology, in cold chain by keeping it in ice packed containers. Each sample was tested for Adenovirus and Entero virus by Polymerase Chain Reaction technique to detect viral DNA. If the sample was negative for both viruses, it was tested for Herpes virus also. Clinical assumption of etiological agent was done. Clinically bacterial conjunctivitis cases were treated with the antibiotic Chloramphenicol eye drops, and clinically viral conjunctivitis cases were treated symptomatically with artificial tear drops. Each patient was followed up on third day, tenth day, and at one month. The patients were examined under slit lamp on each visit \& clinical profile was assessed. Modifications in treatment were given, depending upon the clinical profile and lab results of bacterial culture studies.

\section{Statistical Analysis}

Data was entered in Microsoft Excel and analysed using SPSS 16.0 Categorical variables were summarized as proportions. 
The relationship between selected factors and aetiology was calculated using statistical analysis with chi square test or fisher's exact test.

\section{RESULTS}

\section{Age}

$21 \%$ were in the $1-15$ age group. $51 \%$ were in the $16-50$ age group. And $28 \%$ were in the 51 to 70 years age group.

\section{Clinical Profile}

2 cases presented with complaints of redness on the $1^{\text {st }}$ day of starting of the symptom. 9 cases came on the $2^{\text {nd }}$ day. 14 cases on the third day, 33 cases on the $4^{\text {th }}$ day and 42 cases presented to the outpatient department after 5 days of starting of the symptoms of conjunctivitis.

\section{Type of Discharge at Presentation}

At the time of presentation, $76 \%$ had watery discharge, $12 \%$ had mucoid and another $12 \%$ had purulent discharge. Out of the $23 \%$ patients that turned up for follow up on day 30 , none had any discharge.

\section{Side Affected}

Only 23\% patients turned up for follow up on day $30 \& 77 \%$ were lost follow up. On day 1, 22\% of cases were unilateral, \& $88 \%$ were bilateral. On day $10,1 \%$ were unilateral, $31 \%$ bilateral and $68 \%$ completely recovered. On day 30, only 23 patients came for follow up. Among these 23 patients, 5 had completely recovered and 18 patients showed bilateral involvement (Corneal lesion).

\section{LID Oedema}

On day 1 all patients had lid oedema. On $3^{\text {rd }}$ day $95 \%$ still had lid oedema. On $10^{\text {th }}$ day $18 \%$ cases had lid oedema. Only $23 \%$ patients turned up for follow up on day $30 \& 77 \%$ were lost to follow up. On day $30^{\text {th }}$, none of the cases that turned up for follow up had any lid oedema.

\section{Conjunctival Congestion}

Among the 100 patients involved in the study, all had conjunctival congestion on day l. Out of the 23 patients that came for follow up, none had conjunctival congestion on day 30.

\section{Membranous Reaction}

On the first day only 18 cases out of 100 cases presented with a pseudo membrane. By the $3^{\text {rd }}$ day, it was seen in only 5 cases. This membrane was not seen from the $10^{\text {th }}$ day of follow up in any of the cases. Only $23 \%$ patients turned up for follow up on day $30 \& 77 \%$ were lost to follow up

\section{Sub Conjunctival Haemorrhage}

On the first day $7 \%$ had sub-conjunctival haemorrhage. On day 30 , none had sub-conjunctival haemorrhage.

\section{Chemosis}

On the $1^{\text {st }}$ day, $5 \%$ cases had chemosis. On $3^{\text {rd }}$ day, none of the patients had chemosis. Only $23 \%$ patients turned up for follow up on day $30 \& 77 \%$ were lost to follow up.

\section{Corneal Lesions}

$8 \%$ had corneal involvement on day l which increased to $25 \%$ on day 10. Only 18 cases had corneal lesions at 1 month of follow up. Among the 23\% that turned up for follow up, $78 \%$ had corneal involvement in the form of sub epithelial opacity. Only 23\% patients turned up for follow up on day $30 \& 77 \%$ were lost follow up

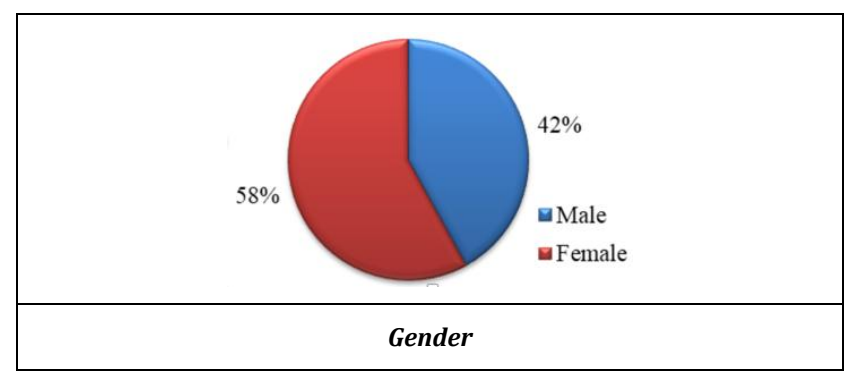

\begin{tabular}{|c|c|c|}
\hline Etiological Agent & Frequency & Percentage \\
\hline Adenovirus & 57 & 57 \\
\hline Enterovirus & 2 & 2 \\
\hline Coagulase negative staphylococci & 19 & 19 \\
\hline Staphylococcus aureus & 1 & 1 \\
\hline Aetiology not found out & 21 & 21 \\
\hline Total & $\mathbf{1 0 0}$ & $\mathbf{1 0 0}$ \\
\hline Aetiological Agent Identified \\
\hline
\end{tabular}

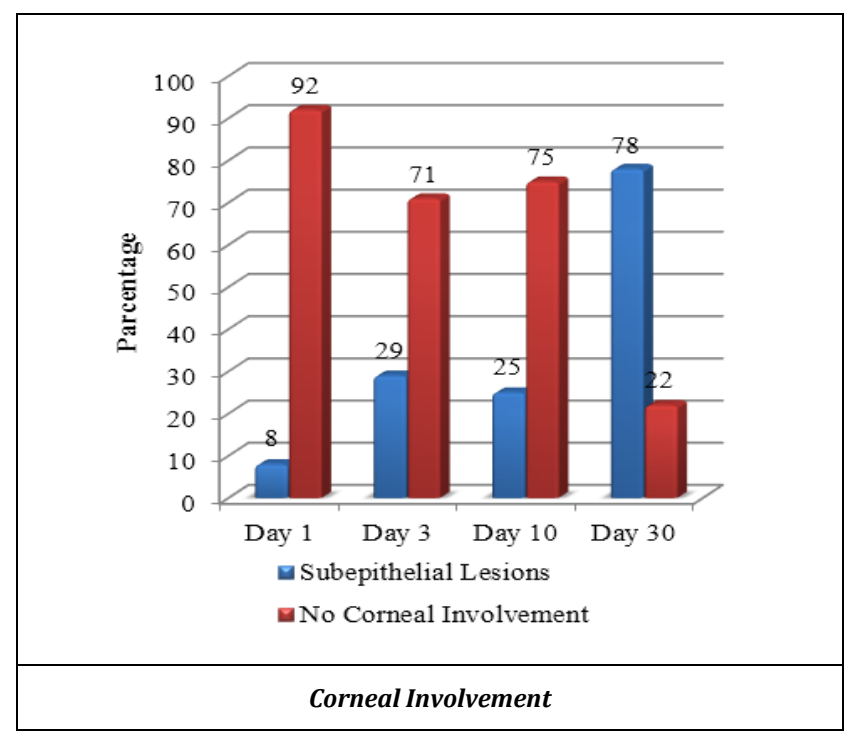

\section{Blurring of Vision}

$97 \%$ of cases had no blurring of vision on the $1^{\text {st }}$ day. $16 \%$ of cases developed blurring of vision from $3^{\text {rd }}$ day. which persisted in 13 cases that turned up for follow up on the $30^{\text {th }}$ day. Only $23 \%$ patients turned up for follow up on day $30 \&$ $77 \%$ were lost follow up

\section{Uveitis}

Only $3 \%$ had uveitis on day l. $1 \%$ had uveitis on day 10. Out of the $23 \%$ patients came for follow up on day 30 , none had uveitis.

\section{Relationship between Age Group and Aetiology}

Viral aetiology was seen in $61.9 \%$ cases in the $1-15$ age group. While $57.1 \%$ of cases in the age group 51 to 70 years had a 
bacterial aetiology. The aetiology could not be found in 22 cases. The differences were not statistically significant with a chi square value 4.572 and a p-value of 0.334 .

\section{Relation between Type of Discharge and Aetiology}

Out of the 100 cases, virus was detected in 54 cases with watery discharge, two cases with mucoid discharge and in 3 cases with purulent discharge. Bacteria were cultured positive or negative in 22 cases with watery discharge, 10 cases with mucoid discharge and in 9 cases with purulent discharge. The differences were statistically significant with chi square value of 19.19 and $p$-value of $<0.001$.

\section{Relation between Type of Conjunctival Reaction and Aetiology}

Follicular reaction was seen 72.45 of cases with viral aetiology. While papillary reaction was seen in 905 cases with bacterial or unknown aetiology. This difference was statistically significant with a chi-square value of 23.710 and a p-value $<0.001$

\section{Lymph Node Enlargement}

Lymph node enlargement was seen in 41 cases of viral conjunctivitis and in 7 cases with bacterial or other conjunctivitis. There was no lymph node enlargement in 18 cases of viral conjunctivitis. The difference was found to be statistically significant with a chi-square value of 26.629 and a p value of $<0.001$.

\section{History of Recent Fever or Upper Respiratory Tract Infection}

$87.5 \%$ of cases with viral aetiology had history of recent fever or exposure to a cases of upper respiratory tract infection. While $67.3 \%$ cases with bacterial conjunctivitis had no such history. The difference was statistically significant with a chi-square value of 30.995 and a $p$ value of $<0.001$.

\begin{tabular}{|c|c|c|c|c|c|}
\hline \multirow{2}{*}{ Aetiology } & \multicolumn{2}{|c|}{ Exposure Present } & \multicolumn{2}{|c|}{ No Exposure } & \multirow{2}{*}{ Total } \\
\cline { 2 - 5 } & Frequency & $\mathbf{\%}$ & Frequency & $\mathbf{\%}$ & \\
\hline Virus & 43 & 67.2 & 16 & 44.6 & 59 \\
\hline Bacteria \& no aetiology & 21 & 32.8 & 20 & 55.4 & 41 \\
\hline Total & $\mathbf{6 4}$ & $\mathbf{1 0 0}$ & $\mathbf{3 6}$ & $\mathbf{1 0 0}$ & $\mathbf{1 0 0}$ \\
\hline \multicolumn{6}{|c|}{ History of Exposure to Conjunctivitis } \\
\hline
\end{tabular}

\section{Corneal Involvement}

Cornea was involved in 4 cases with viral aetiology and in 4 cases with bacterial aetiology on the day of presentation. The difference was not statistically significant with a Fisher's exact test value of 0.713 and $p$-value of 0.589 .

\section{Cornea on Day 10}

Sub epithelial lesions were seen in $86 \%$ of cases with viral aetiology while they were seen in only $14 \%$ cases with bacterial aetiology. The difference was statistically significant with a chi-square value of 8.612 and p-value of 0.003 .

\section{Cornea on Day 30}

Sub epithelial lesions persisted in $94.4 \%$ cases with viral aetiology on the $30^{\text {th }}$ day. While it was seen in only one case with bacterial or unknown aetiology. The difference was statistically significant with a chi-square value of 11.677 and p-value of 0.003 .

\section{Statistical Correlation}

The variables were analysed to check whether they had any statistically significant correlation with the aetiology of conjunctivitis. Variables which had no statistically significant relation to aetiology were gender, duration of symptoms, laterality, corneal lesion on first day, uveitis, chemosis, sub conjunctival haemorrhage, blurring of vision, diabetes mellitus, Immunosuppression, and pre-existing ocular illness. Variables showing statistically significant relation to aetiology were type of discharge, conjunctival reaction, conjunctival membrane, lymph node enlargement, recent history of fever or upper respiratory tract infection and history of exposure to a case of conjunctivitis.

\section{DISCUSSION}

The study identified bacterial etiological agent in $79 \%$ of cases of infectious conjunctivitis by culture, of which $59 \%$ cases were viral positive and $20 \%$ were positive for bacteria. And the etiological agent could not be identified in $21 \%$ of cases.(1) Out of the viral positive cases, $57 \%$ were Adenovirus \& 2\% Enterovirus. Out of the bacteria positive cases, $19 \%$ were Coagulase negative staphylococci \& $1 \%$ was Staphylococcus aureus. These findings are comparable to a similar study by Woodland RM et al in Pakistan.(2) Sociodemographic factors (Age \& sex) didn't have any association to aetiology.(3) History of exposure to conjunctivitis cases within 2 weeks of onset the symptoms, history of recent or concurrent fever or upper respiratory tract infection \& pre auricular or sub mandibular lymph node enlargement, showed statistically significant association with the etiological agent, suggesting a viral aetiology if these parameters were present. Type of discharge showed a statistically significant association with etiological agent suggesting that watery type of discharge was more in favour of a viral aetiology and mucoid or purulent discharge in favour of a bacterial aetiology. These findings were in accordance with the previous studies and existing literature.(4) Conjunctival reaction and type of membrane also showed statistically significant association with etiological agent suggesting that follicular type of conjunctival reaction and conjunctival pseudo membrane favour the diagnosis of viral aetiology for infectious conjunctivitis. These findings were in accordance with the previous studies and existing literature.(5)

Corneal sub- epithelial lesions on day 3, day 10 and day 30 showed a statistically significant association with the etiological agent favouring a diagnosis of viral aetiology if these were present.(6) Blurring of vision on day 3 \& day 10 (As a subjective complaint by the patient) had statistically significant association with the etiological agent suggesting the possibility of virus as the causative organism. However, the blurring of vision was not statistically significant on day $\mathrm{l}$ or day 30. It may be explained as the infective process takes 3-5 days to develop corneal lesions, and by the fact that the lesions tend to resolve after 7-10 days. The day 30 corneal involvement was significant but blurring of vision was not significant, which could be due to clearing of lesions in the central area of the cornea. 


\section{CONCLUSIONS}

The aetiology has no statistically significant relation to sociodemographic factors. The clinical profile of the disease often suggests the etiological agent. Watery discharge, follicular reaction, conjunctival pseudo-membrane, history of recent or concurrent fever or upper respiratory tract infection, pre auricular or sub mandibular lymph node enlargement, \& corneal sub-epithelial lesion favour the probable diagnosis of a viral aetiology. The scientific knowledge about the causative organism and clinical assumption of etiological agent often help in judicious use of antimicrobial agents in infectious conjunctivitis cases. Thus, we can prevent the development of antibiotic resistance developing in the community \& unwanted drug side effects to the patient. The financial burden of treating a very common disease in the community can also be significantly reduced.

\section{Limitations}

Conjunctivitis being a common disease, the sample size was not adequate. The viral detection by PCR studies was expensive. The study didn't include patients without conjunctivitis as controls, so the possibility of isolated organism being a commensal in the conjunctiva could not be ruled out. Only $23 \%$ of the patients turned up for follow up on day $30.77 \%$ of cases were dropouts. So, we could not properly assess the sequelae of infectious conjunctivitis on day 30. The dropouts could be either be due to complete resolution of symptoms or due to the patient approaching another health care facility.

\section{REFERENCES}

[1] Agarwala HS, Raichaudhury M, Munsi NK. Bacterial flora in acute conjunctivitis. J All India Ophthalmol Soc 1967;15(2):58-66.

[2] Woodland RM, Darougar S, Thaker U, et al. Causes of conjunctivitis and keratoconjunctivitis in Karachi, Pakistan. Trans R Soc Trop Med Hyg 1992;86(3):317-20.

[3] O'Brien TP, Jeng BH, McDonald $M$, et al. Acute conjunctivitis: truth and misconceptions. Curr Med Res Opin 2009;25(8):1953-61.

[4] Stenson S, Newman R, Fedukowicz H. Laboratory studies in acute conjunctivitis. Arch Ophthalmol 1982;100(8):1275-7.

[5] Kanski JJ, Bowling B, Nischal $K$, et al. Clinical ophthalmology a systematic approach. $7^{\text {th }}$ edn. Edinburgh: Elsevier 2011: p. 131-52.

[6] Sihota R, Tandon R. Parson's diseases of the eye. $21^{\text {st }}$ edn. New Delhi: Elsevier 2011: p. 161-82. 